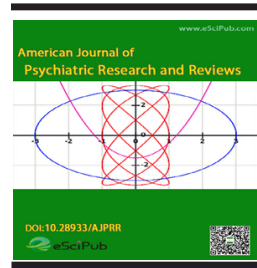

American Journal of Psychiatric Research and Reviews (ISSN:2637-479X)

Post-COVID 19 Experience of Space empty of Matter: Germination of a new science across Zero-Point-Energy

\title{
A.K. Mukhopadhyay
}

All India Institute of Medical Science, New Delhi and North DMC Medical College \& HRH, Delhi

\section{ABSTRACT}

A profound and lasting experience has the power to change the Worldview. For a scientist, this may be the reason for genesis of *Correspondence to Author: a path-making theory. The experience of space empty of matter A.K. Mukhopadhyay by post-COVID19 sufferers, amongst whom are many scientists, All India Institute of Medical Sciis expected to bring zero-point energy (ZPE) state in focus again, Medical College \& $\mathrm{HRH}$, Delhi with the beginning of a new science across ZPE.

Keywords: Post Covid Experience, Space empty of matter, Zero-point-energy

How to cite this article:

A.K.Mukhopadhyay.Post-COVID 19 Experience of Space empty of Matter: Germination of a new science across Zero-Point-Energy. American Journal of Psychiatric Research and Reviews, 2021; 5:27.

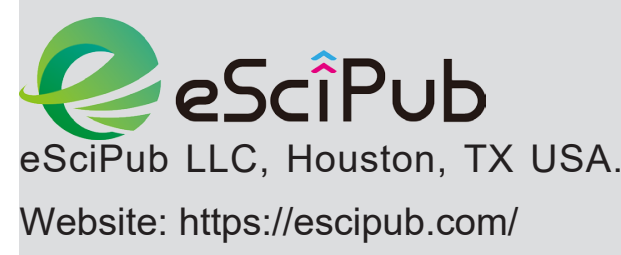


The ongoing COVID-19 pandemic has been seemingly devastating for humanity. However, the silver lining for science is one very common experience by a large number of patients who have recovered from moderate to severe COVID-19 infection. This is the experience of emptiness and nothingness. Amongst these patients are innumerable doctors, psychologists as well as scientists from different disciplines across the continents all over the Globe! I wish, if not all of them but at least the brilliant few ones introspect and analyze this rare experience, the experience becomes relevant for the birth and progress of a post-material new science.

This experience could not be found in DSM-V, which lays down the criteria for diagnosis of psychiatric disorders. This profound feeling of emptiness and nothingness, which is stable and life-changing, merits analysis with the differential diagnosis from depression, anxiety, schizophrenia, and crisis or lysis of existence described by any known terminology. There is no existential issue in this experience, which the subject has already overcome. There is no thanatophobia! There is no disturbing personal integrity. There might be poverty of thoughts, but there is no disorder of thoughts. The mood might be dynamically slow but there is no disorder of mood. The self is stable and situated within the reality. This is a unique post-COVID 19 psychological state of a contentless experience, a kind of emptiness without any substance or matter, the experience that has the life-changing capability. The experience entrains the brain, the heart, and the pulmonary system.

Emptiness is generally described in the context of space while nothingness is in the context of the material. In physics, the matter is that which occupies space and has mass. The post-COVID 19 experience is that of space empty of the matter! This emptiness is felt by the subject within his/her personal space, peri-personal space and global space, which contains absolutely nothing but vacuum without any substance or matter! Worldly signals, which are made of space, time, and energy, which reign in the material plane, are considered of not much use at this stage. Only information prevails within the conscious brain! Signals might be the most important propellers and regulators for lifeless robots. However, information remains the currency, which two conscious entities use for communication. Cognition is the acquisition of information as-such or, information from signals. At this state of emptiness and nothingness, the attention is focussed on "life".

Scientific formulation and theory often germinate from the profound and stable personal experience of the scientist. Einstein discovered the laws of nature on the basis of his profound primary experience. His theory of relativity, and the equation relating mass and energy are based on his unique personal experience. In his life, however, he got stuck with another uncomfortable experience, the experience of space empty of matter. Wisely, he did not develop the concept further and in fact completely banished it from his scientific endeavor fourteen years later he once adopted it $[1,2,3,4]$. This is also widely known as zero-point energy state, and Einstein's cosmological constant.

The energy identified in this space empty of matter is not conventional energy that is related to mass by Einstein's equation. This energy is dark energy widely distributed, and constituting about $70 \%$ of our universe, causatively known for cosmic repulsion events, and expansion of our universe. The human being is generally used to live life within the three-dimensional space with abundant oxygen in the environment. Will not such human beings feel suffocated with threateningly low blood oxygen level, when suddenly pushed into space empty of matter, especially devoid of oxygen?

Associated with this experience of emptiness, extends fortunately a thin web of "life" that spreads all over the fauna and flora of the environment. Amidst the intersections of dark energy and "life", the person feels attracted towards abundance of "life" in nature, in the leafy plants and flowers in the garden, park, roadsides 
and the countryside, and feels as if they too have a language to speak to negotiate with their issues.

What could be the consequences of such an experience of post-COVID 19 emptiness? Not worrisome, for sure! Have trust on our strength of adaptability; neuro-adaptability, neuro-cardiac entrainment, and resilience of our pulmonary system. Rely on life-form's ability to harness dark energy and the movement of various information-states along the ladder of cognition ${ }^{[5]}$. Most of the sufferers would get their emptiness replaced with the richness of life! The environmental fuel in this new growth is the fabrics of "life" while the psychological fuels are faith, devotion, and love ${ }^{[6]}$, with a commitment for the entire ecosystem including humanity. A new humanity has been taking birth across the death tunnel of COVID-19 infection from the state of passive life-less oxygen-inhalation to one step ahead, often-conscious life-full oxygen inhalation. There is every possibility of an emergence of a post-material new science from the experience of space empty of matter following Post-COVID 19 infection.

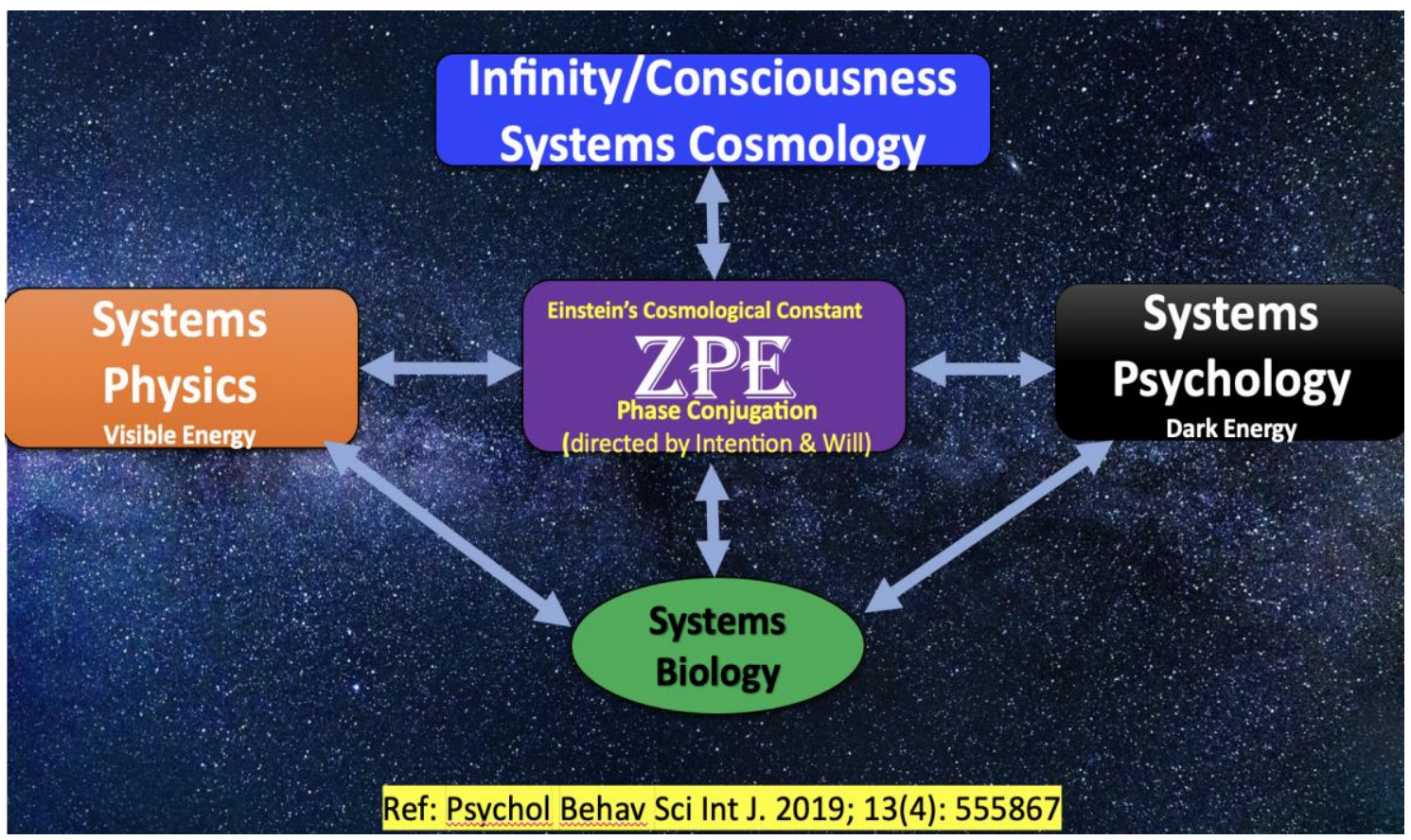

Fig. 1. Space empty of matter or, zero-point energy state is the meeting ground of systems physics, systems psychology, systems cosmology and systems biology.

COVID-19 infection has led the suffering Humanity to an experience of space empty of matter. The result is the emergence of a science across zero-point energy. Zero-point energy state, according to the author, is the meeting room (Figure 1) of physics and psychology, cosmology, and cell biology.

(C) author

\section{References:}

[1]. O'Raifeartaigh, C 2018. Investigating the legend of Einstein's "biggest blunder". Physics Today. 30 Oct. DOI:10.1063/PT.6.3.20181030a

[2]. Gamow G 1956. Sci. Am. 195, 136.
[3]. Taylor EF, Wheeler JA 2000. Exploring Black Holes: Introduction to General Relativity, Addison Wesley.

[4]. Alpher RA1998. Electronic posting on the online message board of the History of Astronomy Discussion Group (HASTRO), 2 April.

[5]. Mukhopadhyay AK, Mukhopadhyay AS 2019. Visualizing Information as a Dynamic Entity Roadmap of Deep Science, Al and Humanity. Psychol Behav Sci Int J.; 13(4): 555867.

[6]. Mukhopadhyay AK 2019. Emergence of Homo spiritualis: Of Essential Requirements - Faith, Devotion and Love: Deep Science of their Psychoneurobiology, Clin Psychiatry 5 (2):62. 